\title{
X-Ray Magnetic Dichroism in the Cobalt-Doped Indium Tin Oxide from First Principle Calculations
}

\author{
V. N. Antonov, ${ }^{1,2}$ L. V. Bekenov, ${ }^{2}$ L. P. Germash, ${ }^{3}$ and N. A. Plotnikov ${ }^{2}$ \\ ${ }^{1}$ Max-Planck-Institut für Festkörperforschung, Heisenberg Straße 1, 70569 Stuttgart, Germany \\ ${ }^{2}$ Institute of Metal Physics, 36 Vernadsky Street, Kiev 03142, Ukraine \\ ${ }^{3}$ National Technical University of Ukraine, Kiev Polytechnical Institute, 14 Politehnichna Street, Kiev 03056, Ukraine
}

Correspondence should be addressed to V. N. Antonov; antonov@imp.kiev.ua

Received 31 May 2013; Accepted 23 September 2013

Academic Editor: Shi J. Xu

Copyright (C) 2013 V. N. Antonov et al. This is an open access article distributed under the Creative Commons Attribution License, which permits unrestricted use, distribution, and reproduction in any medium, provided the original work is properly cited.

\begin{abstract}
The electronic structure of the Co-doped indium tin oxide (ITO) diluted magnetic semiconductors (DMSs) were investigated theoretically from first principles, using the fully relativistic Dirac linear muffin-tin orbital band structure method. The X-ray absorption spectra (XAS) and X-ray magnetic circular dichroism (XMCD) spectra at the Co $L_{3}$, In $M_{2}$, Sn $M_{2}$, and O K edges were investigated theoretically from first principles. The origin of the XMCD spectra in these compounds was examined. The calculated results are compared with available experimental data.
\end{abstract}

\section{Introduction}

Spintronics or spin-transport electronics has attracted much of attention due to its technologically potential applications. Dilute magnetic semiconductors (DMS), obtained by doping a host semiconductor with magnetic impurities, can be used for spintronic devices [1]. In DMS, apart from electron degree of freedom, one uses the spin degree of freedom which can lead to new class of devices and circuits. The starting materials, which were expected to be the promising candidates for spintronics, are Group III-V materials, such as (Ga, Mn)As $[2,3]$ with the Curie temperature of $\sim 110 \mathrm{~K}[4]$. Recently Chen et al. [5] reported the Curie temperature as high as $200 \mathrm{~K}$ in nanostructures of ( $\mathrm{Ga}, \mathrm{Mn})$ As. Other candidates, which can show this property, are transition metal doped Group III nitrides, phosphides, and semiconducting oxides. Dietl et al. [6] predicted theoretically a Curie temperature $T_{C}$ higher than room temperature for transition element doped semiconducting materials, such as $\mathrm{GaN}$ and $\mathrm{ZnO}$. After the report of $T_{C} \sim 280 \mathrm{~K}$ in $\left(\mathrm{Zn}_{1-x} \mathrm{Co}_{x}\right) \mathrm{O}$ [7], there have been many reports on ZnO-based DMSs showing high $T_{C}$ [8]. Both theoretical and experimental studies suggested that wide bandgap oxide semiconductors with high-carrier density are one of the most favorable host compounds for ferromagnetic
DMS with higher Curie temperature [9-12]. Compared to nonoxide semiconductors, the advantages of oxide semiconductors are (1) wide bandgap suited for applications with short wavelength light, (2) transparency and dyeability with pigments, (3) high n-type carrier concentration, (4) capability to be grown at low temperature even on plastic substrate, (5) ecological safety and durability, (6) low cost, and so forth. In addition, large electronegativity of oxygen is expected to produce strong $p-d$ exchange coupling between band carriers and localized spins. Such advantages make oxide semiconductors attractive. Actually, many studies on oxidebased DMS have been reported [13], where most of the research employed $\mathrm{ZnO}$ and $\mathrm{TiO}_{2}$ as host semiconductors.

To integrate electronics, magnetics, and photonics for next generation multifunctional devices, it will be important to search for a magnetic semiconductor with tunable carrier density, high mobility, and magnetic moment as well as optical transparency. $\operatorname{In}_{2} \mathrm{O}_{3}$ is a very promising candidate for this task. It is a well-known semiconducting oxide already in practical use worldwide in batteries, transparent infrared reflectors, and photovoltaics. Indium oxide has a cubic bixbyite structure with a lattice parameter of $10.118 \AA$ [14, 15] and a direct bandgap of $3.75 \mathrm{eV}$. Films of $\mathrm{In}_{2} \mathrm{O}_{3}$ are superior to other transparent conductors largely due to their 
higher mobility $\left(10-75 \mathrm{~cm}^{2} \mathrm{~V}^{-1} \mathrm{~s}^{-1}\right.$ at a carrier density of $\sim 10^{19}-10^{20} \mathrm{~cm}^{-3}$ ) and effective mass of $0.3 m_{e}$ [16]. When $\mathrm{In}_{2} \mathrm{O}_{3}$ is doped with $\mathrm{SnO}_{2}$, the so-called indium tin oxide (ITO) is formed. Similar to its parent indium oxide, ITO is generally nonstoichiometric with respect to oxygen. It shows a rather high electrical conductivity, arising from a large electron concentration, which follows from doping with Sn and from the presence of oxygen vacancies. Since it exhibits technologically important properties, ITO is a widely studied material [16-18]. In recent years, transitional element doped ITO magnetic semiconductors are extensively studied due to their potential applications in spintronic devices [19-32].

Philip et al. [19] have reported high temperature ferromagnetism in Mn doped ITO thin films. Hong et al. [25] have undertaken a systematic investigation on structural and magnetic properties of $\mathrm{V} / \mathrm{Cr} / \mathrm{Fe} / \mathrm{Co} / \mathrm{Ni}$-doped $\operatorname{In}_{2} \mathrm{O}_{3}$ thin films grown on $\mathrm{MgO}$ and $\mathrm{Al}_{2} \mathrm{O}_{3}$ substrates with $5 \% \mathrm{TM}$ concentration. All of the TM: $\operatorname{In}_{2} \mathrm{O}_{3}$ films were found to be ferromagnetic above room temperature. As for $\mathrm{Fe}$ and $\mathrm{Co}$ doping, the saturation magnetization $\left(M_{s}\right)$ was very modest (0.4 and $0.5 \mu_{\mathrm{B}} /$ atom, resp.). As for $\mathrm{Ni}$ doping, the value of $M_{s}$ of the Ni: $\operatorname{In}_{2} \mathrm{O}_{3}$ film was $0.7 \mu_{\mathrm{B}} /$ atom. The authors concluded that the room temperature ferromagnetism (FM) in $\mathrm{V} / \mathrm{Cr} / \mathrm{Fe} / \mathrm{Co} / \mathrm{Ni}$-doped $\mathrm{In}_{2} \mathrm{O}_{3}$ films probably stems from the doped $\mathrm{In}_{2} \mathrm{O}_{3}$ matrices. Kim et al. [28] reported on the structural, magnetic, and magnetotransport characteristics of Cr-doped indium tin oxide films. Room temperature ferromagnetism was observed for $5 \mathrm{~mol} \% \mathrm{Cr}$-doped ITO. They presented clear evidence of room temperature ferromagnetism, exchange interaction between itinerant carriers and localized $\mathrm{Cr}$ spins, and anomalous Hall effect governed by electron doping in laser-deposited Cr-doped ITO films. The intrinsic nature of carrier-mediated ferromagnetism, optical transparency, and semiconducting behavior with high-electron concentration and low resistivity in Cr-doped ITO could represent a promising application for transparent semiconductor spin electronics devices operable at room temperature.

There is yet no consensus on how ferromagnetism comes about in DMS oxides. It is clear that growth processes and postgrowth annealing strongly affect their properties. However, experimental results conducted on both bulks and thin ITO films by different groups sometimes are quite different and even contradictory. He et al. [20] and Yoo et al. [21] reported high temperature ferromagnetism in Fe-doped or $\mathrm{Fe}, \mathrm{Cu}$-codoped $\mathrm{In}_{2} \mathrm{O}_{3}$ films, while Peleckis et al. [29] found only paramagnetism in Fe-doped $\operatorname{In}_{2} \mathrm{O}_{3}$. In addition, Kohiki et al. [22] and Ohno et al. [23] attributed the room temperature $\mathrm{FM}$ to the formation of $\mathrm{Fe}_{2} \mathrm{O}_{3}$ nanoclusters dispersed in $\operatorname{In}_{2} \mathrm{O}_{3}$ matrix. On the other hand, Xing et al. [31] observed high temperature ferromagnetism and perpendicular magnetic anisotropy in Fe-doped $\operatorname{In}_{2} \mathrm{O}_{3}$ magnetic semiconductor films with a Curie temperature as high as $927 \mathrm{~K}$. Extensive microstructure, composition, and magnetic studies indicate that the Fe element incorporates into the indium oxide lattice by substituting the position of indium atoms, which suggests that the observed ferromagnetism is intrinsic rather than from the Fe clusters or any other magnetic impurity phases.
The origin of ferromagnetism in ITO:Co also remains unclear. Nearly all published papers on this system report similar magnetic and transport results. However, the interpretations are often controversial. In some cases, hightemperature annealing of epitaxial ITO:Co films grown by pulsed laser deposition seems to remove the metallic Co nanoclusters, existing in as-grown films, and incorporate magnetic ions into the host matrix, which leads to intrinsic ferromagnetism. On the other hand, it has more recently been found that annealing, while removing metallic Co nanoclusters from the bulk, promotes their diffusion to the interface between the substrate and the film in the same system [30]. Consequently, the observed ferromagnetism in annealed films arises from Co particles. Hong et al. [26] obtained room temperature ferromagnetism in Co-doped $\mathrm{In}_{2} \mathrm{O}_{3}$ thin films grown on sapphire and $\mathrm{MgO}$ substrates. Under various conditions, ferromagnetic Co: $\mathrm{In}_{2} \mathrm{O}_{3}$ films were well crystallized as $\operatorname{In}_{2} \mathrm{O}_{3}$ structure and no trace of any secondary phase could be seen in the spectra. Co atoms were perfectly substituted for In atoms, and their distribution in the $\mathrm{In}_{2} \mathrm{O}_{3}$ host matrix was greatly uniform. The films grown at a rather low temperature as of $550^{\circ} \mathrm{C}$ have the largest magnetic moment of about $0.5 \mu_{\mathrm{B}} /$ Co. A change of the substrate temperature of $50^{\circ} \mathrm{C}$ could change the magnetization by one order of magnitude. Stankiewicz et al. [30] show that the magnetic and electrical transport properties of indium tin oxide thin films doped with Co can be varied in a wide range by postgrowth annealing in an oxidizing, inert, or reducing atmosphere. ITO films with up to 8 at.\% of Co seem to be well substituted and show intrinsic FM behavior even above $400 \mathrm{~K}$. They found that the spin magnetic moment/Co of these films correlates with electron (donor) concentration $n$ and varies from 0.1 to $1.0 \mu_{\mathrm{B}}$ with $n$ changing from $10^{19}$ to $10^{21} \mathrm{~cm}^{-1}$. The magnetic moment per Co ion also strongly depends on the Co concentration. The films with more than 8 at. \% of Co show rather high values of the magnetic moment per Co ion $\left(\sim 1.7 \mu_{\mathrm{B}}\right)$. Much smaller magnetic moments were obtained for homogeneous ferromagnetic ITO:Co films with less than 8 at.\% of Co. The authors claim a major role of oxygen vacancies in establishing ferromagnetic coupling in these materials. However, they found no clear correlation between the values of the magnetic moment and the number of oxygen vacancies in the ITO films.

The major interest of previous investigations was concentrated on the nature of the magnetic interactions in the DMSs. In the present study, we focus our attention on X-ray absorption spectra (XAS) as well as X-ray magnetic circular dichroism (XMCD) in Co-doped ITO DMSs. The XMCD experiments measure the difference of the absorption of $\mathrm{X}$ rays with opposite (left and right) directions of circular polarization. The XMCD is a powerful tool to study the elementspecific local magnetic interactions and also it reflects the spin and orbital polarizations of the local electronic states. The XAS and XMCD at the Co $L_{2,3}$, In, and Sn $M_{2}$ edges in the Co doped ITO were measured by Hakimi et al. [32]. They also calculated the dichroism spectra at the Co $L_{3}$ edge using atomic multiplet calculations with some adjustable parameters. The results of Hakimi et al. [32] suggest that the Co ions are substituted for the In site in ITO. Magnetic field 
and temperature-dependent XMCD spectra imply that the Co ions give a paramagnetic contribution to the overall ferromagnetic response. No XMCD could be detected at the In $M_{2}$ or Sn $M_{2}$ edge, thus excluding the presence of a large magnetic polarization of the In and Sn sublattices. They suggest that the ferromagnetism in Co-doped ITO is not related to the $3 d$ electronic states. These findings may point towards the likelihood that the bulk ferromagnetism observed from the SQUID magnetometry measurements may be oxygen mediated, for example, oxygen vacancies or oxygen-induced defects. The authors concluded that further studies are required to definitively observe dichroism at oxygen sites at remanence. This might confirm the presence of a spontaneous ferromagnetic contribution.

In this work we present first principles calculations of the electronic structure, XAS and XMCD spectra at the Co $L_{2,3}$, In and $\operatorname{Sn} M_{2,3}$, and $\mathrm{O} K$ edges in the Co doped ITO. We also investigated the influence of the oxygen vacancies on the electronic and magnetic structures of the host ITO and ITO:Co DMSs. The paper is organized as follows. Section 2 presents structural models for the Co doped ITO DMSs and the details of the calculations. Section 3 is devoted to the electronic structure as well as XAS and XMCD properties of Co doped ITO calculated with the fully relativistic Dirac LMTO band structure method. The results are compared with available experimental data. Finally, the results are summarized in Section 4.

\section{Computational Details}

The elements in the ITO:Co compound have nominal atomic structures $[\mathrm{Kr}] 4 d^{10} 5 s^{2} 5 p^{1}$ for In, $[\mathrm{Kr}] 4 d^{10} 5 s^{2} 5 p^{2}$ for $\mathrm{Sn}$, [Ar] $3 d^{7} 4 s^{2}$ for Co, and $[\mathrm{He}] 2 s^{2} 2 p^{4}$ for O. The most stable and therefore most common position of Co in the ITO host lattice is at the In site where its two $4 s$ electrons can participate in crystal bonding in much the same way as the two In $5 \mathrm{~s}$ electrons.

The calculations of the electronic structure of the ITO:Co DMSs were performed for supercells of $\operatorname{In}_{2} \mathrm{O}_{3}$ cubic bixbyite structure unit cell with one In ion replaced by Co. The supercell calculations were performed for the $\left(\mathrm{In}_{0.80} \mathrm{Sn}_{0.20}\right)_{2(1-x)} \mathrm{Co}_{2 x} \mathrm{O}_{3}$ compound with composition $x=$ 0.0625 using the $I A \overline{3}$ (Number 206) space group. The substitutional ITO:Co positions are illustrated in Figure 1 for a 40atom ITO unit cell containing one substitutional Co atom. The Co atom has six $\mathrm{O}$ nearest neighbors at the distance of $2.1973 \AA$. The second-neighbor shell consists of 12 In atoms: six at the distance of $3.3571 \AA$ and six at the distance $3.8324 \AA$.

The details of the computational method are described in our previous papers [33-35], and here we only mention some aspects specific to the present calculations. The calculations presented in this work were performed using the spin-polarized fully relativistic linear muffin-tin orbital (SPR LMTO) method $[36,37]$ for the experimentally observed lattice constants $a=10.145 \AA$ for the $\operatorname{In}_{2} \mathrm{O}_{3}$ [38]. The crystal structure was optimized using the Vienna ab initio simulation package (VASP) [39-41]. For LMTO calculations we used the Perdew-Wang [42] parameterization of the exchangecorrelation potential. Brillouin zone (BZ) integrations were

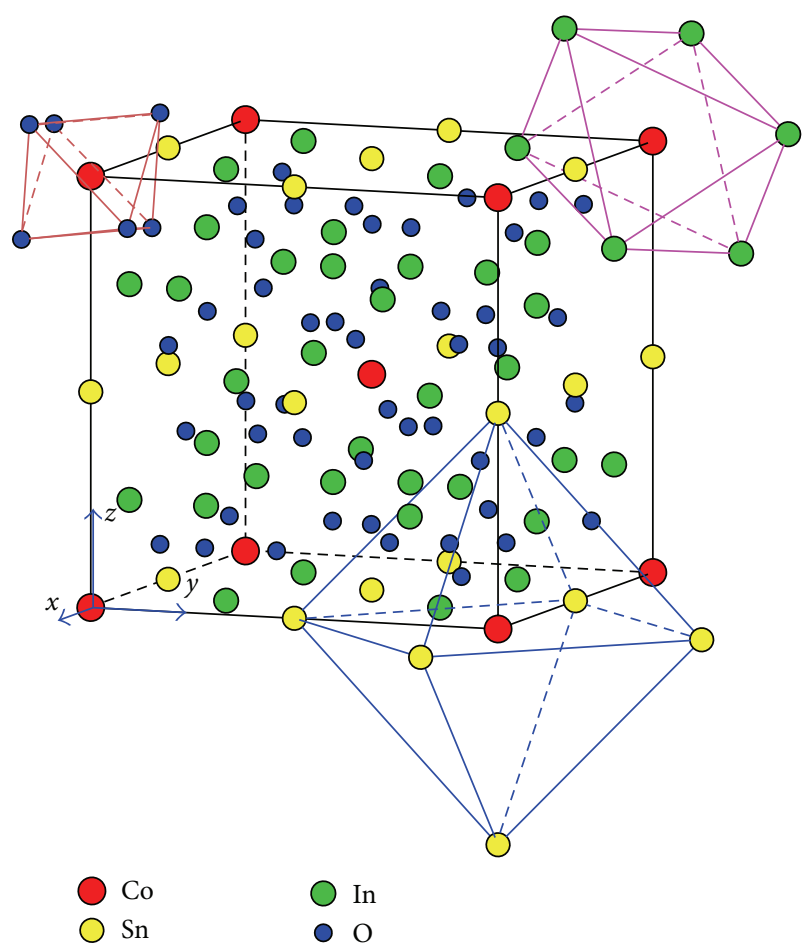

FIGURE 1: Schematic representation of a 40-atom Co-doped ITO $\left(\mathrm{In}_{0.80} \mathrm{Sn}_{0.20}\right)_{2(1-x)} \mathrm{Co}_{2 x} \mathrm{O}_{3}$.

performed using the improved tetrahedron method [43] and charge self-consistency was obtained on a grid of $294 \mathbf{k}$ points in the irreducible part of the BZ of the ITO:Co. To improve the potential we included additional interstitial spheres. The basis consisted of Co, In, $\mathrm{Sn}$ and $\mathrm{O} s, p$, and $d$ linear muffintin orbitals, the basis for empty spheres consisted of $s$, and $p$ LMTOs.

The X-ray absorption and dichroism spectra were calculated taking into account the exchange splitting of core levels. The finite lifetime of a core hole was accounted for by folding the spectra with a Lorentzian. The widths of the core level spectra, $\Gamma_{L_{2}}=1.13 \mathrm{eV}, \Gamma_{L_{3}}=0.47 \mathrm{eV}$ for Co; $\Gamma_{M_{2}}=2.7 \mathrm{eV}$ for In; $\Gamma_{M_{2}}=2.85 \mathrm{eV}$ for $\mathrm{Sn}$, and $\Gamma_{K}=0.18 \mathrm{eV}$ for O; were taken from [44]. The finite apparative resolution of the spectrometer was accounted for by a Gaussian of width $0.6 \mathrm{eV}$.

Strong electronic correlations in Co $3 d$ shell were treated at the mean-field level using the LSDA $+U$ approach [45] in its rotationally invariant implementation which is described in detail in our previous paper [46]. The effective on-site Coulomb repulsion $U$ was treated as an adjustable parameter. We used $U=4 \mathrm{eV}$ for the Co $3 d$ states. The exchange integral $J$ was estimated from constrained LSDA calculations, and values of $0.92 \mathrm{eV}$ were used.

\section{Results and Discussion}

3.1. Energy Band Structure. Figure 2(a) presents $\mathrm{Co}_{d}$ partial density of states (PDOS) for a 40-atom $\left(\mathrm{In}_{0.80} \mathrm{Sn}_{0.20}\right)_{2(1-x)} \mathrm{Co}_{2 x} \mathrm{O}_{3}$ (ITO) unit cell containing one $\mathrm{Co}_{\text {In }}$ substitution $(x=0.0625)$ in the LSDA approximation. 


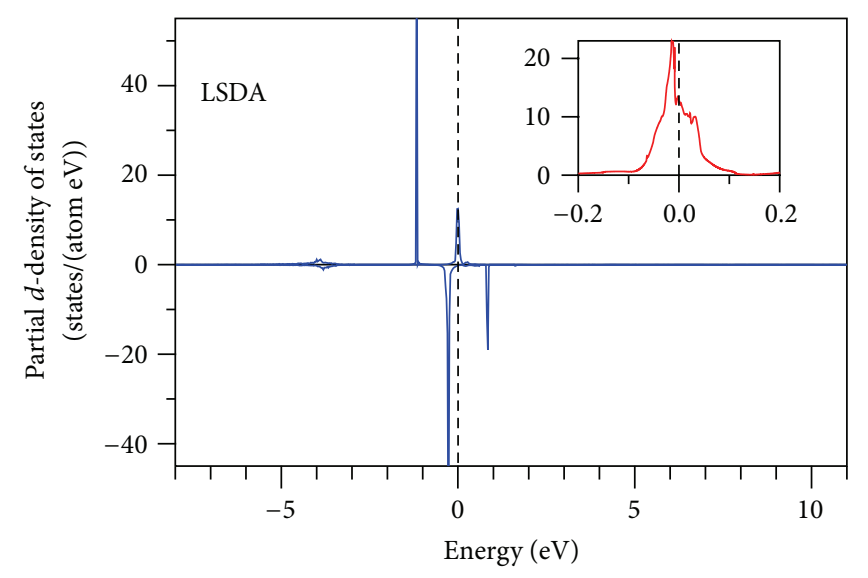

(a)

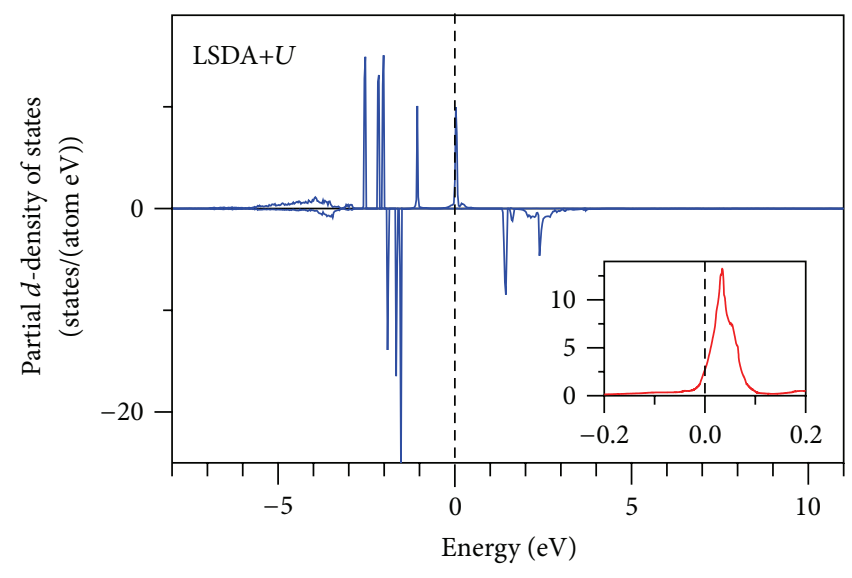

(b)

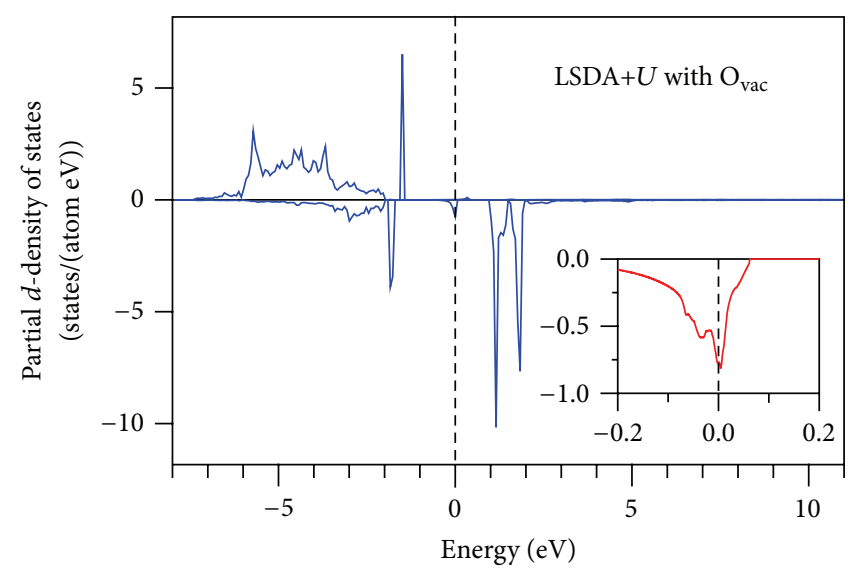

(c)

FIgURE 2: $\mathrm{Co}_{\text {In }}$ substitution $3 d$ partial density of states [in states/(atom eV)] ions in ITO calculated in the LSDA approximation (a), the $L S D A+U$ approach (b), and the LSDA $+U$ approach with oxygen vacancy in close vicinity to Co ion (c). The insert is a blowup of the Co $d$ PDOS close to the Fermi energy. The Fermi energy is at zero.

There is a strong narrow peak in the PDOS situated at the Fermi level. It indicates the strong Coulomb correlations in the Co $3 d$ shell. To describe correctly localized Co $3 d$ states in ITO:Co we used the LSDA+U approach [46]. The correspondent PDOS is presented in Figure 2(b). The Hubbard $U_{\text {eff }}$ strongly affects the energy distribution of the Co $3 d$ states shifting occupied states downwards by the $U_{\text {eff }} / 2$ and empty $3 d$ states upwards by the same amount.

Figure 3 presents total and partial density of states for In, $\mathrm{Sn}, \mathrm{O}$, and Co sites in ITO:Co unit cell in the LSDA+U approach. The $\mathrm{O} 2 s$ states are located mostly between -20.6 and $-17.7 \mathrm{eV}$ below the Fermi level and the $2 p$ states of the $\mathrm{O}$ are found between $-9.7 \mathrm{eV}$ and $-2.8 \mathrm{eV}$. The spin splitting of the $\mathrm{O} p$ states is quite small. The In $5 s$ states are located between -8.5 and $-4.5 \mathrm{eV}$ below the Fermi level and from $1.5 \mathrm{eV}$ to $7.9 \mathrm{eV}$ above $\varepsilon_{F}$. The $5 p$ states of the In are found between $-8.5 \mathrm{eV}$ and $-3.0 \mathrm{eV}$ and from $3.1 \mathrm{eV}$ to $14.2 \mathrm{eV}$. The Sn $5 s$ states are presented as quite narrow peaks at -9.2 to $-9.7 \mathrm{eV}$ below $\varepsilon_{F}$ which is weakly hybridized with Co and In states. There is also a narrow peak in close vicinity of the $\varepsilon_{F}$ from $-1.0 \mathrm{eV}$ to $0.8 \mathrm{eV}$ due to hybridization with the Co $3 d$ states. The Sn $5 p$ states are located between $-8.5 \mathrm{eV}$ and $-4.0 \mathrm{eV}$ and from $1.8 \mathrm{eV}$ to $11.0 \mathrm{eV}$ above $\varepsilon_{F}$. The Co $3 d$ impurity states hybridize well with the $\mathrm{O} 2 p$ conduction band, which gives the solution a metallic character. We also observe quite strong In $5 s-S n 5 p$ hybridization at $1.5 \mathrm{eV}$ to $4.5 \mathrm{eV}$ above $\varepsilon_{F}$. The crystal field at the $\mathrm{Co}_{\text {In }}$ site $\left(S_{6}\right.$ point symmetry) causes the splitting of Co $d$ orbitals into a singlet $a_{g}$ (the linear combination of the $d_{x y}, d_{y z}$, and $d_{x z}$ ) and two doublets $e_{g}\left(d_{3 z^{2}-1}\right.$ and $\left.d_{x^{2}-y^{2}}\right)$ and $e_{g 1}\left(d_{x y}, d_{y z}\right.$ and $\left.d_{x z}\right)$. A very strong and narrow peak of the majority-spin $\operatorname{Co} d_{y z}$ and $d_{x z}$ symmetry is found in close vicinity to the Fermi energy at around $0.03 \mathrm{eV}$ above the Fermi level (see the insert in Figure 3(e)).

The magnetic moment in the ITO:Co unit cell is $1.160 \mu_{\mathrm{B}}$ with Co spin magnetic moment of $1.150 \mu_{\mathrm{B}}$ in the $\left(\mathrm{In}_{0.80} \mathrm{Sn}_{0.20}\right)_{2(1-x)} \mathrm{Co}_{2 x} \mathrm{O}_{3}(x=0.06)$. The orbital moment at the Co site is rather small, of about $0.040 \mu_{\mathrm{B}}$. The induced spin magnetic moments at the $\mathrm{O}$ first neighbor sites ranged from $-0.002 \mu_{\mathrm{B}}$ to $0.001 \mu_{\mathrm{B}}$ for different oxygen sites. We obtained rather small spin magnetic moments around $0.006 \mu_{\mathrm{B}}$ for $\mathrm{Sn}$ and $0.002 \mu_{\mathrm{B}}$ for In mostly coming from the $p$ valence states. 


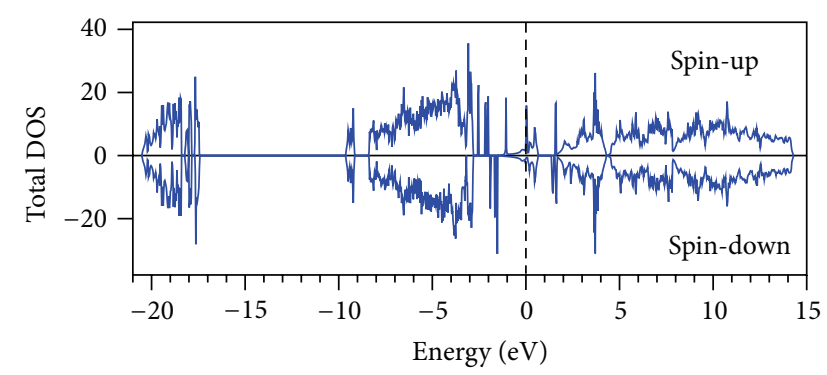

(a)

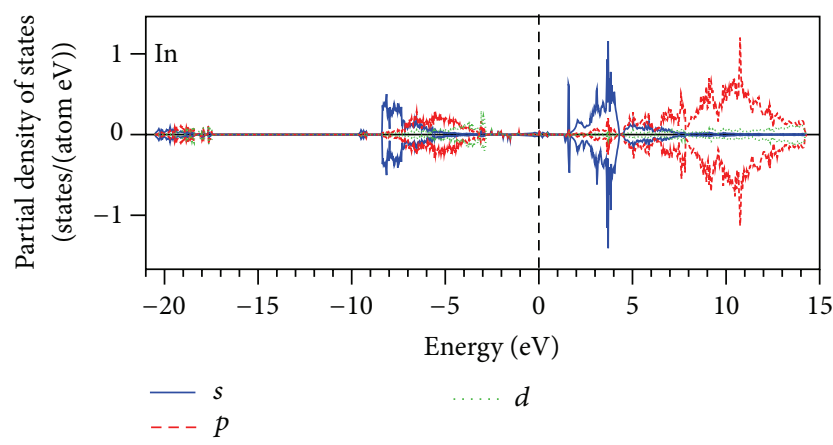

(c)

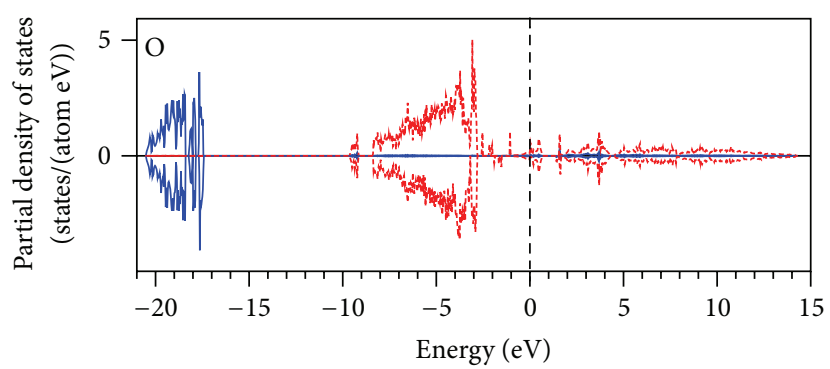

$-s$

$--p$

(b)

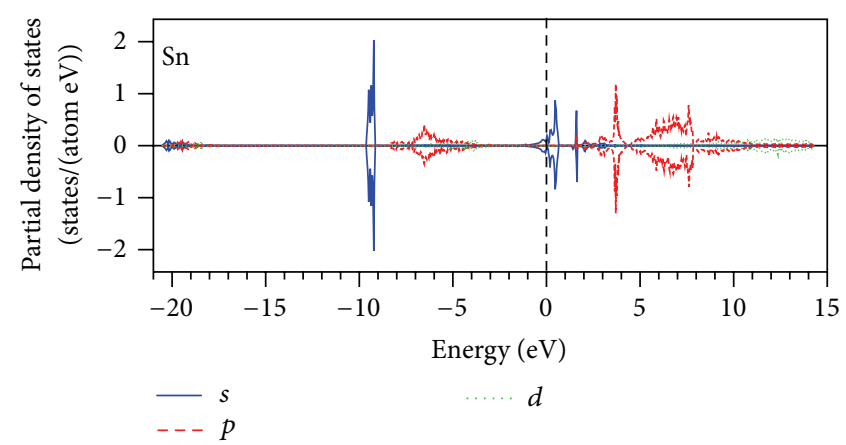

(d)

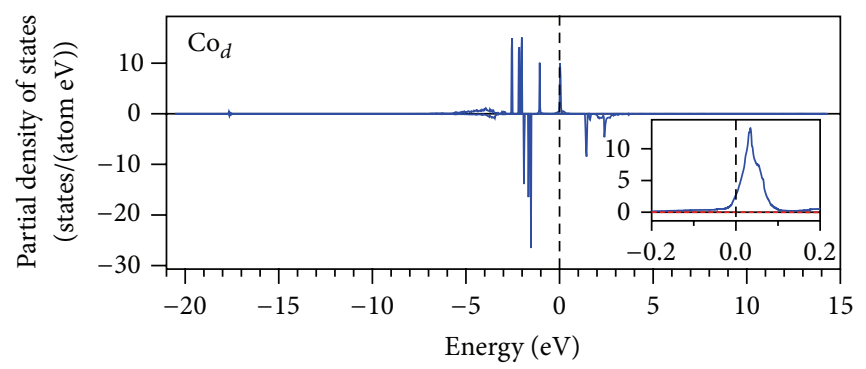

(e)

Figure 3: The $\mathrm{LSDA}+U$ total [in states/(cell eV)] and partial [in states/(atom eV)] densities of states for the $\mathrm{O}$, In, $\mathrm{Sn}$, and substitutional $\mathrm{Co}_{\text {In }}$ ions in ITO. The insert is a blowup of the Co $d$ PDOS close to the Fermi energy. The Fermi energy is at zero.

The orbital moments were found to be extremely small, less than $10^{-4} \mu_{\mathrm{B}}$ for both the $\mathrm{Sn}$ and In ions. The orbital moment at the $\mathrm{O}$ site is larger, of about $0.0003 \mu_{\mathrm{B}}$.

It is well-known that defects appear to play a crucial role in the development of ferromagnetism in DMSs [47]. However, the exact role and contribution to the underlying mechanism of the defects in stabilizing ferromagnetism are yet to be established. Defects may include oxygen and cationic vacancies, as well as interstitial ions.

To investigate the influence of possible oxygen vacancies on the electronic structure of the ITO:Co DMSs we create an oxygen vacancy in the first neighborhood of the cobalt ion. The oxygen vacancy strongly affects the energy distribution of the Co $3 d$ partial DOSs in close vicinity to the Fermi level for the relaxed lattice (compare in Figures 2(b) and 2(c)). The oxygen vacancy also changes the spin and orbital moments at the $\mathrm{Co}$ and $\mathrm{O}$ sites. The spin and orbital magnetic moments at the Co site are increased up to $2.619 \mu_{\mathrm{B}}$ and $0.071 \mu_{\mathrm{B}}$, respectively. The spin magnetic moments at the $\mathrm{O}$ sites are increased by one order of magnitude in comparison with the ideal ITO:Co crystal without oxygen vacancies and range from $-0.031 \mu_{\mathrm{B}}$ to $0.046 \mu_{\mathrm{B}}$ for different oxygen sites. Orbital moments at the $\mathrm{O}$ site with oxygen vacancy are increased by approximately $10 \%$ in comparison with the ideal ITO:Co lattice.

3.2. X-Ray Absorption and XMCD Spectra. In order to investigate the possibility of magnetic polarization at the In and Sn lattice sites, XAS and XMCD spectra were recorded by Hakimi et al. [32] at the In and Sn $M_{2}$ edges at $2 \mathrm{~K}$ under an applied magnetic field of $4 \mathrm{~T}$. No evidence of dichroic behavior at the In $M_{2}$ and Sn $M_{2}$ edges was detected experimentally. Figures 4 and 5 show the experimental XAS and XMCD spectra together with the spectra calculated in the $\mathrm{LSDA}+U$ approach. The shapes of the In $M_{2}$ and $\mathrm{Sn} M_{2} \mathrm{X}-$ ray absorption spectra are well reproduced by the theory (Figures 4(a) and 5(a)). Both $\mathrm{In}^{3+}$ and $\mathrm{Sn}^{4+}$ have an electronic configuration of $4 d^{10}$ and one would not expect any magnetic 


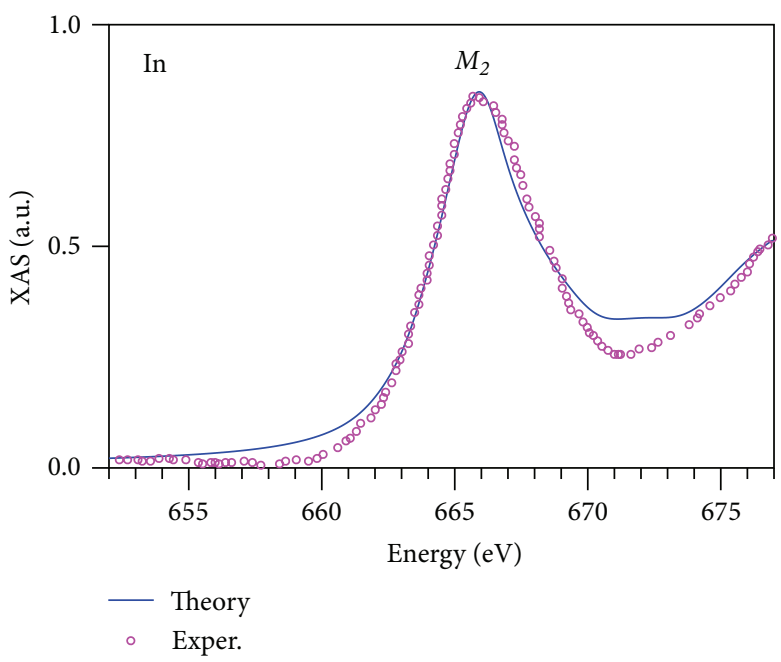

(a)

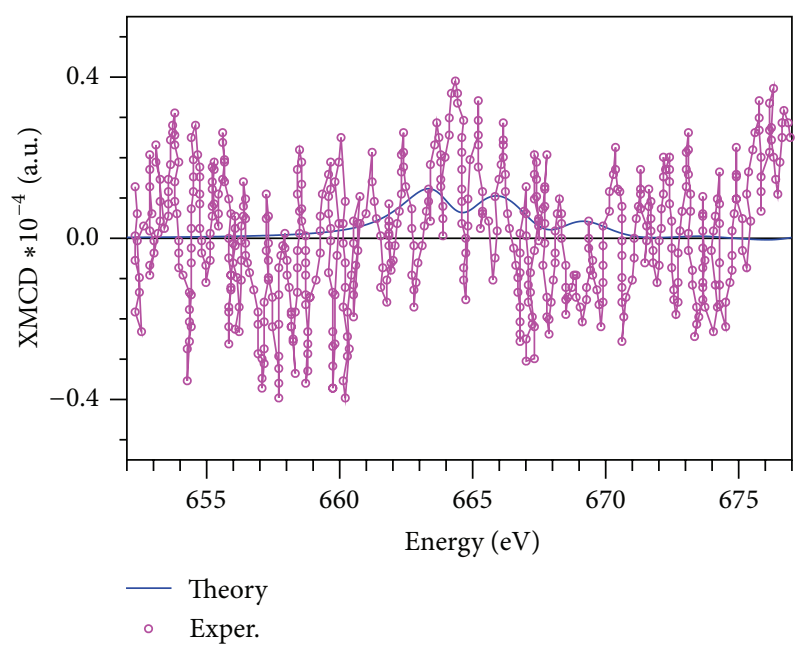

(b)

Figure 4: X-ray absorption (a) and XMCD (b) experimental spectra [32] (circles) of ITO:Co at the In $M_{2}$ edge measured at $2 \mathrm{~K}$ under an applied field of $4 \mathrm{~T}$ and theoretically calculated spectra (full blue lines).

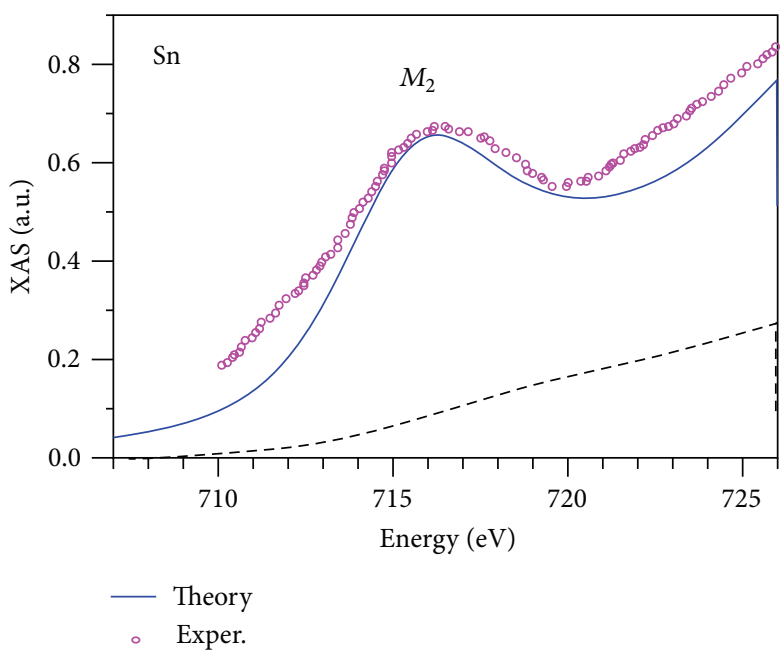

(a)

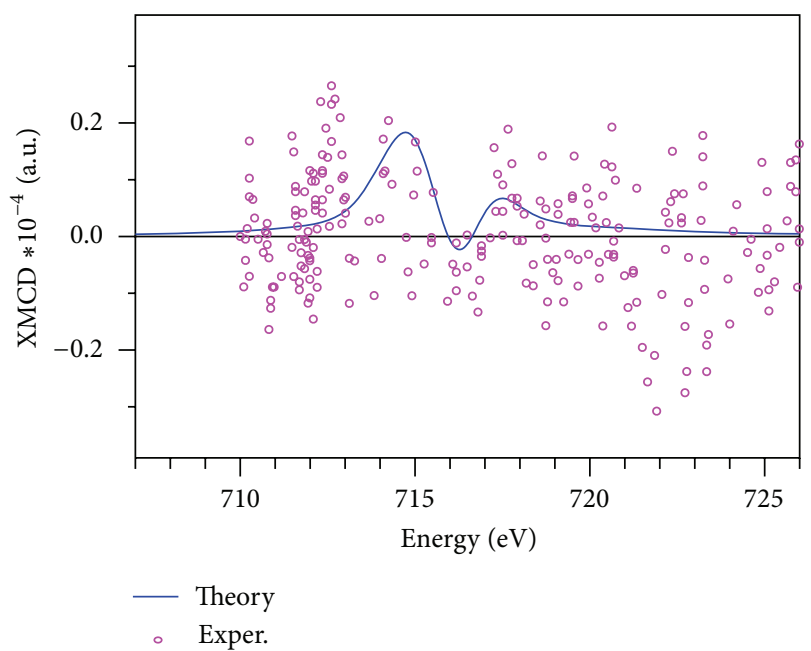

(b)

FIGURE 5: X-ray absorption (a) and XMCD (b) experimental spectra [32] (circles) of ITO:Co at the Sn $M_{2}$ edge measured at $2 \mathrm{~K}$ under an applied field of $4 \mathrm{~T}$ and theoretically calculated spectra (full blue lines).

moment to be present at all. However, we obtained in the LSDA $+U$ calculations small spin magnetic moments around $0.006 \mu_{\mathrm{B}}$ for Sn and $0.002 \mu_{\mathrm{B}}$ for In mostly coming from the $p$ valence states. The orbital moments were found to be extremely small, less than $10^{-4} \mu_{\mathrm{B}}$ for both the Sn and In ions. As a result, the theoretically calculated X-ray magnetic dichroism at the In $M_{2}$ and $\operatorname{Sn} M_{2}$ edges was found to be very small-four orders of magnitude smaller than the value of the corresponding absorption. The predicted magnetic moments for In and Sn are extremely small, considerably less than the limit of detection using XMCD (around $0.01 \mu_{\mathrm{B}}$ ); therefore Hakimi et al. [32] were not able to see any dichroism at the In and $\mathrm{Sn} M_{3}$ edges (Figures 4 and 5).
Figure 6 presents experimental XAS and XMCD spectra [32] of Co doped ITO at the Co $L_{3}$ edge together with the spectra calculated in the $\mathrm{LSDA}+U$ approach. The $\mathrm{X}$-ray absorption spectrum at the Co $L_{3}$ edge is rather complicated and consists of two major structures: major peaks $b$ and $c$ at around $774.5 \mathrm{eV}$ and $775.6 \mathrm{eV}$, respectively, with a low energy shoulder $a$ at $773.6 \mathrm{eV}$, peak $d$ at $776.3 \mathrm{eV}$, and a high energy fine structure $e$ at $778 \mathrm{eV}$.

Two theoretical results are presented in Figure 6: without any additional defects (full blue lines) and with the oxygen vacancy (red dashed lines). As may be seen from Figure 6(B) the calculations for the ideal crystal structure with one substitutional $\mathrm{Co}_{1}$ atom provide the $\mathrm{X}$-ray absorption intensity 


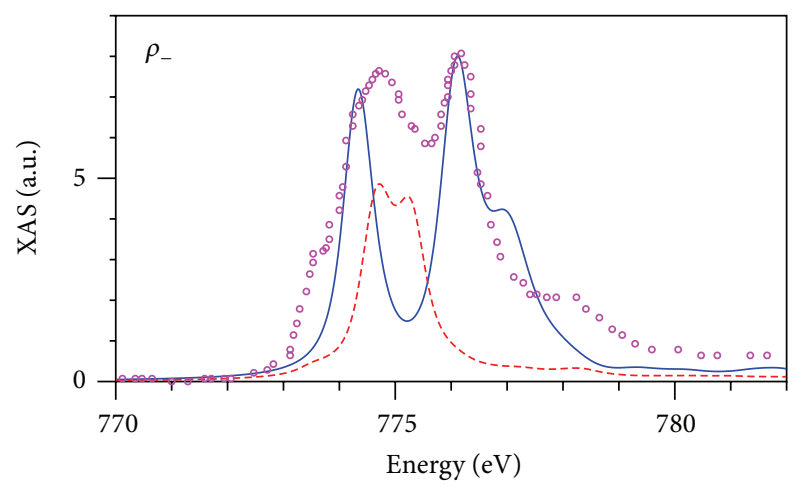

(A)

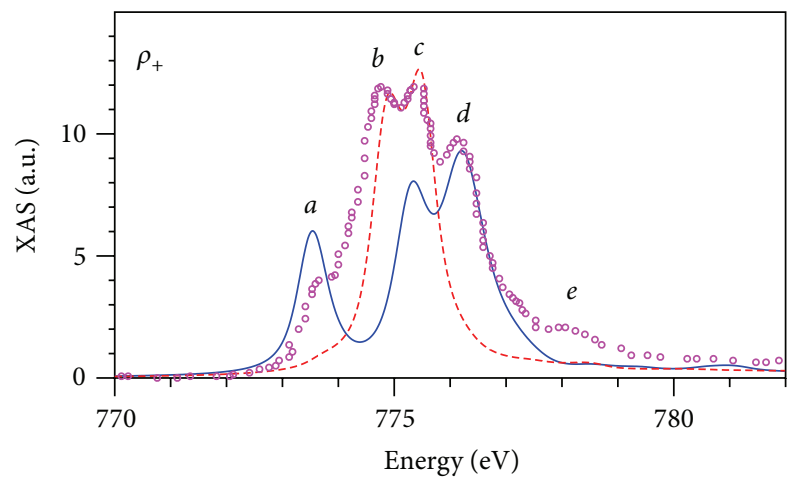

(B)

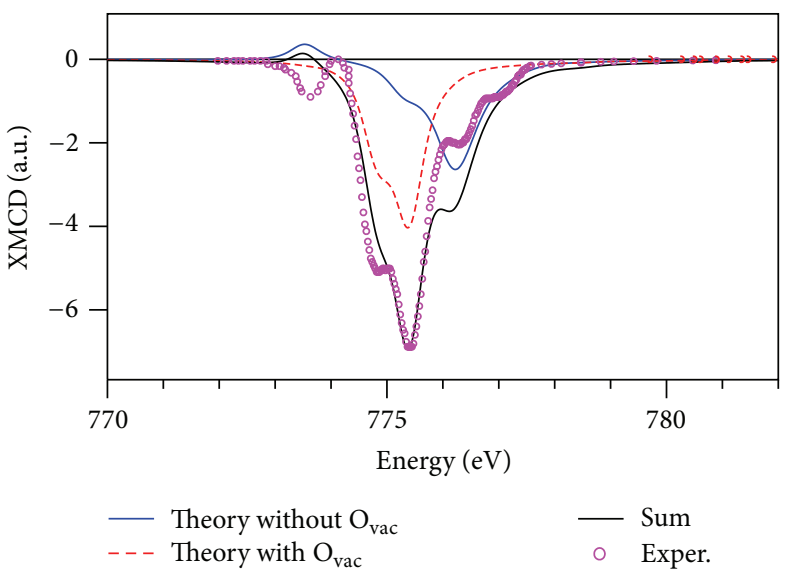

(C)

FIGURE 6: X-ray absorption experimental spectra [32] (circles) of ITO:Co at the Co $L_{3}$ edge for left $\left(\rho_{-}\right)(\mathrm{A})$ and right $\left(\rho_{+}\right)$(B) circularly polarized light measured at $2 \mathrm{~K}$ under an applied field of $4 \mathrm{~T}$ in comparison with the theoretically calculated XAS spectra for the $\mathrm{Co}_{1}$ without any additional defects (full blue lines) and $\mathrm{Co}_{2}$ with the oxygen vacancy ions (dashed red lines); (C) experimentally measured XMCD spectra [32] (circles) and theoretically calculated ones for the $\mathrm{Co}_{1}$ without any additional defects (full blue lines) and $\mathrm{Co}_{2}$ with the oxygen vacancy ions (dashed red lines).

for right $\left(\rho_{+}\right)$circularly polarized light only at the peaks $a$, $c$, and $d$. The full explanation of the spectra is only possible by taking crystal imperfections such as oxygen deficiency into account. The oxygen vacancy (with lattice relaxation taken into account) strongly affects the Co $3 d$ partial density of state (Figure 2(c)) and the shape of the XAS spectra. The X-ray absorption from the $\mathrm{Co}_{2}$ atoms with the oxygen vacancy in close vicinity mostly determine the shape of the major double peak $b$ and $c$. Our calculations including oxygen deficiency reproduce the shape of the Co $L_{3} \mathrm{X}$ ray absorption spectra quite well for both the right and left circularly polarized lights except for the high energy structure $e$ which is not reproduced by the theoretical calculations. It might be that the additional satellite structure at the 


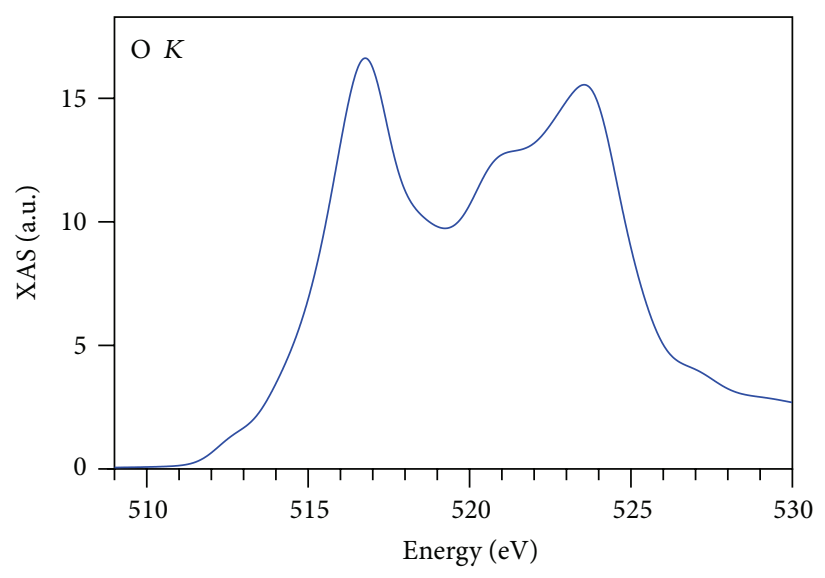

(a)

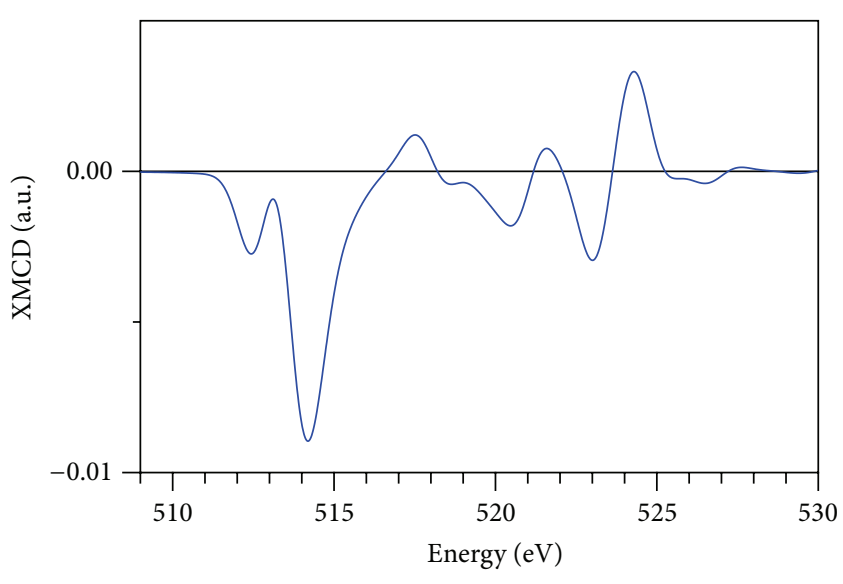

(b)

FIgURE 7: The theoretically calculated X-ray absorption (a) and XMCD (b) spectra of Co doped ITO at the O $K$ edge.

high energy tail of the Co $L_{3}$ XAS appears due to manybody effects. This question needs an additional theoretical investigation using an appropriate many-body treatment.

The theoretically calculated Co $L_{3}$ XMCD spectrum is in good agreement with the experiment (full black curve in Figure 6(C)). The major minimum at around $775.6 \mathrm{eV}$ was found to be due to both contributions with and without the oxygen vacancy in the Co doped ITO DMS. The theory does not produce the fine structure corresponding to the high energy satellite structure $e$ at around $778 \mathrm{eV}$. Also, the experimentally measured small negative peak at $773.6 \mathrm{eV}$ has a positive sign in theory.

To investigate the magnetization in the Co doped ITO Hakimi et al. [32] used the SQUID magnetometry and XMCD measurements. They found that in the SQUID magnetometry measurements the magnetic hysteresis loop shows a curve with saturation field of approximately $0.4-0.5 \mathrm{~T}$ with a large coercive field. On the other hand, the XMCD magnetization curve only shows signs of saturation beyond the measurement range of $4 \mathrm{~T}$. Although the XMCD measurements in applied magnetic field of $4 \mathrm{~T}$ at temperature of $2 \mathrm{~K}$ show quite intensive dichroic signal at the Co $L_{2,3}$ edges, the dichroism at remanence (the magnetization left behind after the external magnetic field was removed) was extremely small. It can be pointed toward paramagnetic behavior of Co ions in ITO at least at $2 \mathrm{~K}$, thus excluding their significant contribution to the ferromagnetism. The authors conclude that the Co ions are not directly responsible for the ferromagnetism observed in the Co doped ITO films and the bulk ferromagnetism observed from the SQUID magnetometry measurements may be oxygen mediated, for example, oxygen vacancies or oxygen-induced defects. This is rather contradictory to what has been assumed to account for the magnetic properties in other DMS systems such as transition metal doped GaAs $[2,3,35]$ or $\mathrm{ZnO}[8,48-50]$.

In our theoretical calculations we found the ferromagnetic solution in Co doped ITO with a quite large spin and orbital magnetic moments. On the other hand, to obtain the shape of the XAS and XMCD spectra at the Co $L_{2,3}$ edges we have to take into account the oxygen vacancies. Therefore, lattice imperfections such as oxygen vacancies play important role in magnetic structure of Co doped ITO DMS and oxygen $K$ edge XAS data can certainly be capable to provide a useful information on whether the features could be signatures of oxygen related lattice defects.

Unfortunately, Hakimi et al. [32] did not present the experimental XAS and XMCD spectra at the oxygen $K$ edge; therefore Figure 7 shows only the theoretically calculated spectra. The $\mathrm{O} K$ edge spectra fundamentally reveal a transition from the $\mathrm{O} 1 s$ core states to the unoccupied $\mathrm{O} 2 p$ derived states, which are hybridized with the relatively narrow $3 d$ band and broader $4 s p$ bands of the Co ions. We obtained relatively large dichroism at the oxygen $K$ edge which is two orders of magnitude larger than it was observed at the In and Sn $L_{2,3}$ edges.

To confirm the presence of a spontaneous ferromagnetic contribution in the presence of oxygen vacancies we fulfilled the band structure and XMCD calculations for pure $\operatorname{In}_{2} \mathrm{O}_{3}$. We obtained a nonmagnetic semiconductor solution for $\mathrm{In}_{2} \mathrm{O}_{3}$. On the other hand, we found that the oxygen vacancy can strongly polarize the lattice. The average spin magnetic moment at the oxygen was found to be equal to $0.09 \mu_{\mathrm{B}}$. For several separate oxygen sites the spin and orbital magnetic moments reach the value of $0.22 \mu_{\mathrm{B}}$ and $0.002 \mu_{\mathrm{B}}$, respectively. We also obtained a well-pronounced dichroism spectrum at the oxygen $K$ edge without any Co impurities. We can conclude that the ferromagnetism in Co doped ITO probably originated from both the Co impurities as well as oxygen vacancies. The experimental investigation of the magnetic structure and XMCD properties in Co doped and pure ITO with different levels of the oxygen vacancies is highly desired to make the final answer on the ferromagnetic nature of the Co doped ITO.

\section{Summary}

We have studied the electronic structure and X-ray magnetic circular dichroism of the Co doped ITO diluted magnetic semiconductors by means of $a b$ initio fully relativistic spin-polarized Dirac linear muffin-tin orbital method. The 
absorption and the dichroism spectra at the $\mathrm{O} K$, Co $L_{3}$, In, and $\mathrm{Sn} L_{2}$ edges were investigated.

The theoretical calculations show very small dichroism signals at the In $M_{2}$ and Sn $M_{2}$ edges. The shape of the In $M_{2}$ and $\mathrm{Sn} M_{2} \mathrm{X}$-ray absorption spectra are well reproduced by the theory.

The theory reproduces the shape and energy positions of major fine structures of the Co XAS and XMCD spectra at the $L_{3}$ edge in ITO:Co DMS reasonably well. We show that oxygen deficiency is responsible for some fine structures of the Co $L_{3}$ XAS and XMCD spectra.

We obtained relatively large dichroism at the oxygen $K$ edge which is two orders of magnitude larger than it was observed at the In and Sn $L_{2,3}$ edges. We found that the oxygen vacancy can strongly polarize the lattice. The average spin magnetic moment at the oxygen was found to be equal to $0.09 \mu_{\mathrm{B}}$. For several separate oxygen sites the spin and orbital magnetic moments at the oxygen sites reach the value of $0.22 \mu_{\mathrm{B}}$ and $0.002 \mu_{\mathrm{B}}$, respectively. The experimental investigation of magnetic structure and XMCD properties in Co doped and pure ITO with different level of the oxygen vacancies is highly desired to get the final answer on the ferromagnetic nature of the Co doped ITO.

\section{Acknowledgments}

This work was supported by the National Academy of Sciences of Ukraine in the framework of the State Target Scientific and Technology Program "Nanotechnology and Nanomaterials" for 2010-2014 (no. 0277092303) and Implementation and Application of Grid Technologies for 2009-2013 (no. 0274092303). V. N. Antonov gratefully acknowledges the hospitality at Max-Planck-Institut für Festkörperforschung in Stuttgart during his stay there. The third author of the paper L. P. Germash unfortunately recently passed away.

\section{References}

[1] J. K. Furdyna, "Diluted magnetic semiconductors," Journal of Applied Physics, vol. 64, no. 4, pp. R29-R64, 1988.

[2] S. Sanvito, G. Theurich, and N. A. Hill, "Density functional calculations for III-V diluted ferromagnetic semiconductors: a review," Journal of Superconductivity and Novel Magnetism, vol. 15, no. 1, pp. 85-104, 2002.

[3] T. Jungwirth, J. Sinova, J. Mašek, J. Kučera, and A. H. MacDonald, "Theory of ferromagnetic (III, Mn) V semiconductors," Reviews of Modern Physics, vol. 78, no. 3, pp. 809-864, 2006.

[4] H. Ohno, A. Shen, F. Matsukura et al., "(Ga,Mn)As: a new diluted magnetic semiconductor based on GaAs," Applied Physics Letters, vol. 69, no. 3, pp. 363-365, 1996.

[5] L. Chen, X. Yang, F. Yang et al., "Enhancing the curie temperature of ferromagnetic semiconductor (Ga, Mn)As to $200 \mathrm{~K}$ via nanostructure engineering," Nano Letters, vol. 11, no. 7, pp. 2584-2589, 2011.

[6] T. Dietl, H. Ohno, F. Matsukura, J. Cibert, and D. Ferrand, "Zener model description of ferromagnetism in zinc-blende magnetic semiconductors," Science, vol. 287, no. 5455, pp. 10191022, 2000.
[7] K. Ueda, H. Tabata, and T. Kawai, "Magnetic and electric properties of transition-metal-doped $\mathrm{ZnO}$ films," Applied Physics Letters, vol. 79, no. 7, pp. 988-990, 2001.

[8] C. Liu, F. Yun, and H. Morko, "Ferromagnetism of ZnO And GaN: a review," Journal of Materials Science, vol. 16, no. 9, pp. 555-597, 2005.

[9] D. D. Awschalom and R. K. Kawakami, “Teaching magnets new tricks," Nature, vol. 408, no. 6815, pp. 923-924, 2000.

[10] S. J. Pearton, C. R. Abernathy, M. E. Overberg et al., "Wide band gap ferromagnetic semiconductors and oxides," Journal of Applied Physics, vol. 93, no. 1, pp. 1-13, 2003.

[11] W. Prellier, A. Fouchet, and B. Mercey, "Oxide-diluted magnetic semiconductors: a review of the experimental status," Journal of Physics, vol. 15, no. 37, pp. R1583-R1601, 2003.

[12] S. J. Pearton, W. H. Heo, M. Ivill, D. P. Norton, and T. Steiner, "Dilute magnetic semiconducting oxides," Semiconductor Science and Technology, vol. 19, no. 10, pp. R59-R74, 2004.

[13] T. Fukumura, Y. Yamada, H. Toyosaki, T. Hasegawa, H. Koinuma, and M. Kawasaki, "Exploration of oxide-based diluted magnetic semiconductors toward transparent spintronics," Applied Surface Science, vol. 223, no. 1-3, pp. 62-67, 2004.

[14] R. D. Shannon, J. L. Gillson, and R. J. Bouchard, "Single crystal synthesis and electrical properties of $\mathrm{CdSnO}_{3}, \mathrm{Cd}_{2} \mathrm{SnO}_{4}$, $\mathrm{In}_{2} \mathrm{TeO}_{6}$ and $\mathrm{Cdln}_{2} 2 \mathrm{O}_{4}$," Journal of Physics and Chemistry of Solids, vol. 38, no. 8, pp. 877-881, 1977.

[15] P. Nath and R. F. Bunshah, "Preparation of $\operatorname{In}_{2} \mathrm{O}_{3}$ and tindoped $\mathrm{In}_{2} \mathrm{O}_{3}$ films by a novel activated reactive evaporation technique," Thin Solid Films, vol. 69, no. 1, pp. 63-68, 1980.

[16] K. L. Chopra, S. Major, and D. K. Pandya, "Transparent conductors: a status review," Thin Solid Films, vol. 102, no. 1, pp. $1-46,1983$.

[17] I. Hamberg and C. G. Granqvist, "Evaporated Sn-doped $\mathrm{In}_{2} \mathrm{O}_{3}$ films: basic optical properties and applications to energyefficient windows," Journal of Applied Physics, vol. 60, no. 11, article R123, 1986.

[18] H. L. Hartnagel, A. L. Dawar, A. K. Jain, and C. Jagadish, Semiconducting Transparent Thin Films, Institute of Physics, Bristol, UK, 1995.

[19] J. Philip, N. Theodoropoulou, G. Berera, J. S. Moodera, and B. Satpati, "High-temperature ferromagnetism in manganesedoped indium-tin oxide films," Applied Physics Letters, vol. 85, no. 5, pp. 777-779, 2004.

[20] J. He, S. Xu, Y. K. Yoo et al., "Room temperature ferromagnetic n-type semiconductor in $\left(\operatorname{In}_{1-x} \mathrm{Fe}_{x}\right)_{2} \mathrm{O}_{3-\sigma}$," Applied Physics Letters, vol. 86, no. 5, Article ID 052503, 2005.

[21] Y. K. Yoo, Q. Xue, H. C. Lee et al., "Bulk synthesis and hightemperature ferromagnetism of $\left(\mathrm{In}_{1-x} \mathrm{Fe}_{x}\right)_{2} \mathrm{O}_{3-\sigma}$ with $\mathrm{Cu}$ codoping," Applied Physics Letters, vol. 86, no. 4, Article ID 042506, 2005.

[22] S. Kohiki, Y. Murakawa, K. Hori et al., "Magnetic behavior of Fe-doped $\mathrm{In}_{2} \mathrm{O}_{3}$, Japanese Journal of Applied Physics, vol. 44, no. 28-32, pp. L979-L981, 2005.

[23] T. Ohno, T. Kawahara, H. Tanaka et al., "Ferromagnetism in transparent thin films of Fe-doped indium tin oxide," Japanese Journal of Applied Physics, vol. 45, no. 33-36, pp. L957-L959, 2006.

[24] N. H. Hong, J. Sakai, N. T. Huong, and V. Brize, "Room temperature ferromagnetism in laser ablated Ni-doped $\operatorname{In}_{2} \mathrm{O}_{3}$ thin films ", Applied Physics Letters, vol. 87, no. 10, Article ID 102505, 2005. 
[25] N. H. Hong, J. Sakai, N. T. Huong, A. Ruyter, and V. Brize, "Magnetism in transition-metal-doped $\mathrm{In}_{2} \mathrm{O}_{3}$ thin films," Journal of Physics, vol. 18, no. 29, Article ID 6897, 2006.

[26] N. H. Hong, J. Sakai, N. T. Huong, and V. Brize, "Co-doped $\mathrm{In}_{2} \mathrm{O}_{3}$ thin films: room temperature ferromagnets," Journal of Magnetism and Magnetic Materials, vol. 302, no. 1, pp. 228-231, 2006.

[27] J. Philip, A. Punnoose, B. I. Kim et al., "Carrier-controlled ferromagnetism in transparent oxide semiconductors," Nature Materials, vol. 5, no. 4, pp. 298-304, 2006.

[28] H. S. Kim, S. H. Ji, H. Kim et al., "Observation of ferromagnetism and anomalous Hall effect in laser-deposited chromiumdoped indium tin oxide films," Solid State Communications, vol. 137, no. 1-2, pp. 41-43, 2006.

[29] G. Peleckis, X. L. Wang, and S. X. Dou, "Room-temperature ferromagnetism in Mn and Fe codoped," Applied Physics Letters, vol. 88, no. 13, Article ID 132507, 2006.

[30] J. Stankiewicz, F. Villuendas, and J. Bartolome, "Magnetic behavior of sputtered Co-doped indium-tin oxide films," Physical Review B, vol. 75, no. 23, Article ID 235308, 2007.

[31] P. F. Xing, Y. X. Chen, S. S. Yan et al., "High temperature ferromagnetism and perpendicular magnetic anisotropy in $\mathrm{Fe}-$ doped $\mathrm{In}_{2} \mathrm{O}_{3}$ films," Applied Physics Letters, vol. 92, no. 2, Article ID 022513, 2008.

[32] A. M. H. R. Hakimi, F. Schoofs, R. Bali et al., "Origin of magnetism in cobalt-doped indium tin oxide thin films," Physical Review B, vol. 82, no. 14, Article ID 144429, 2010.

[33] V. N. Antonov, H. A. Dürr, Y. Kucherenko, L. V. Bekenov, and A. N. Yaresko, "Theoretical study of the electronic and magnetic structures of the Heusler alloys $\mathrm{Co}_{2} \mathrm{Cr}_{1-x} \mathrm{Fe}_{x} \mathrm{Al}$," Physical Review B, vol. 72, no. 5, Article ID 054441, 2005.

[34] V. N. Antonov, O. Jepsen, A. N. Yaresko, and A. P. Shpak, "Electronic structure and X-ray magnetic circular dichroism in the Heusler alloy $\mathrm{Co}_{2} \mathrm{MnGe}$," Journal of Applied Physics, vol. 100, no. 4, Article ID 043711, 2006.

[35] V. N. Antonov, A. N. Yaresko, and O. Jepsen, "X-ray magnetic dichroism in III-V diluted magnetic semiconductors: firstprinciples calculations," Physical Review B, vol. 81, no. 7, Article ID 075209, 2010.

[36] O. K. Andersen, "Linear methods in band theory," Physical Review B, vol. 12, no. 8, pp. 3060-3083, 1975.

[37] V. V. Nemoshkalenko, A. E. Krasovskii, V. N. Antonov et al., "Relativistic linear muffin-tin orbital method: application to Au," Physica Status Solidi B , vol. 120, no. 1, pp. 283-296, 1983.

[38] S. Saib and N. Bouarissa, "Structural phase transformations of GaN and InN under high pressure," Physica B, vol. 387, no. 1-2, pp. 377-382, 2007.

[39] P. E. Blöchl, "Projector augmented-wave method," Physical Review B, vol. 50, no. 24, pp. 17953-17979, 1994.

[40] J. P. Perdew, K. Burke, and M. Ernzerhof, "Generalized gradient approximation made simple," Physical Review Letters, vol. 78, no. 7, pp. 1396-1396, 1997.

[41] G. Kresse and J. Joubert, "From ultrasoft pseudopotentials to the projector augmented-wave method," Physical Review B, vol. 59, no. 3, pp. 1758-1775, 1999.

[42] J. P. Perdew and Y. Wang, "Accurate and simple analytic representation of the electron-gas correlation energy," Physical Review B, vol. 45, no. 23, pp. 13244-13249, 1992.

[43] P. E. Blöchl, O. Jepsen, and O. K. Andersen, "Improved tetrahedron method for Brillouin-zone integrations," Physical Review $B$, vol. 49, no. 23, pp. 16223-16233, 1994.
[44] J. L. Campbell and T. Parr, "Widths of the atomic K-N $\mathrm{N}_{7}$ levels," Atomic Data and Nuclear Data Tables, vol. 77, no. 1, pp. 1-56, 2001.

[45] V. I. Anisimov, J. Zaanen, and O. K. Andersen, "Band theory and Mott insulators: hubbard U instead of Stoner I," Physical Review B, vol. 44, no. 3, pp. 943-954, 1991.

[46] A. N. Yaresko, V. N. Antonov, and P. Fulde, "Localized U $5 f$ electrons in $\mathrm{UPd}_{3}$ from $\mathrm{LDA}+\mathrm{U}$ calculations," Physical Review $B$, vol. 67, no. 15, pp. 155103-155114, 2003.

[47] B. Ali, L. R. Shah, C. Ni, J. Q. Xiao, and S. I. Shah, "Interplay of dopant, defects and electronic structure in driving ferromagnetism in Co-doped oxides: $\mathrm{TiO}_{2}, \mathrm{CeO}_{2}$ and $\mathrm{ZnO}$," Journal of Physics, vol. 21, no. 45, Article ID 456005, 2009.

[48] L. V. Bekenov, V. N. Antonov, S. Ostanin et al., "Electronic and magnetic properties of $\left(\mathrm{Zn}_{1-x} \mathrm{~V}_{x}\right) \mathrm{O}$ diluted magnetic semiconductors elucidated from $\mathrm{X}$-ray magnetic circular dichroism at $\mathrm{v}$ $\mathrm{L}_{2,3}$ edges and first-principles calculations," Physical Review B Condensed Matter and Materials Physics, vol. 84, no. 13, Article ID 134421, 2011.

[49] V. N. Antonov, L. V. Bekenov, O. Jepsen, D. V. Mazur, and L. P. Germash, "X-ray magnetic dichroism in the (Zn, Co)O diluted magnetic semiconductors from first principle calculations," Journal of Applied Physics, vol. 111, no. 7, Article ID 073702, 2012.

[50] V. N. Antonov, L. V. Bekenov, D. V. Mazur, and L. P. Germash, "X-ray magnetic dichroism in $(\mathrm{Zn}, \mathrm{Mn}) \mathrm{O}$ diluted magnetic semiconductors: first-principles calculations," Journal of the Korean Physical Society, vol. 60, no. 11, pp. 1915-1922, 2012. 

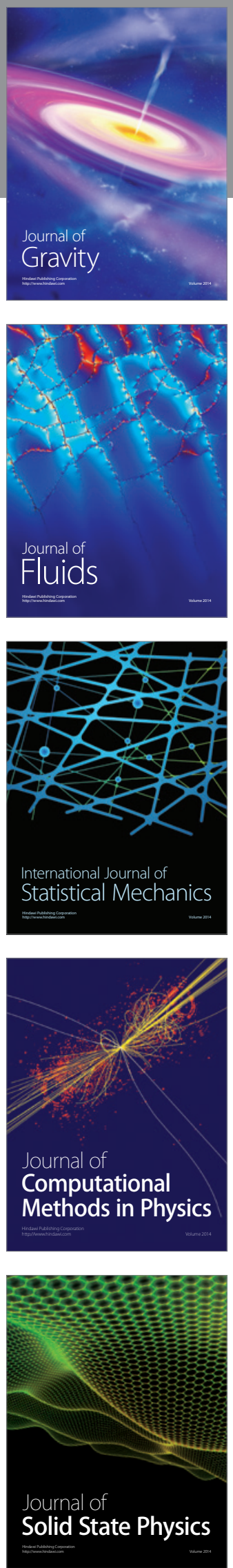

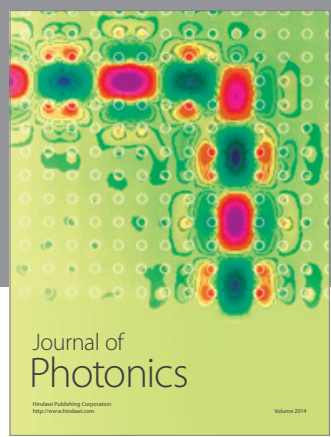

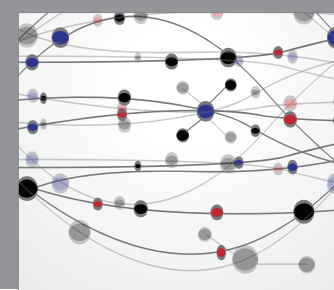

The Scientific World Journal

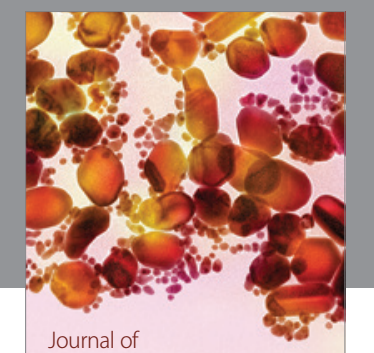

Soft Matter
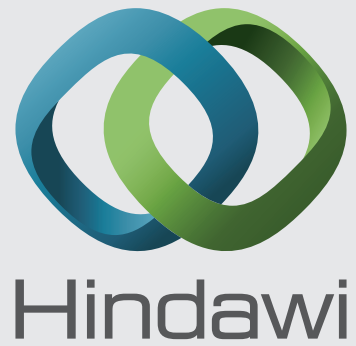

Submit your manuscripts at

http://www.hindawi.com
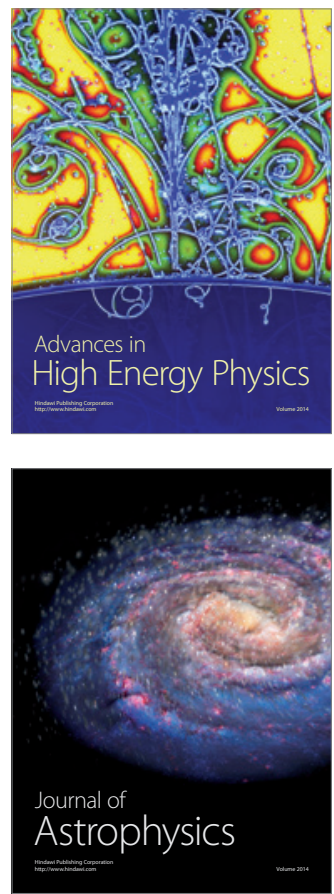
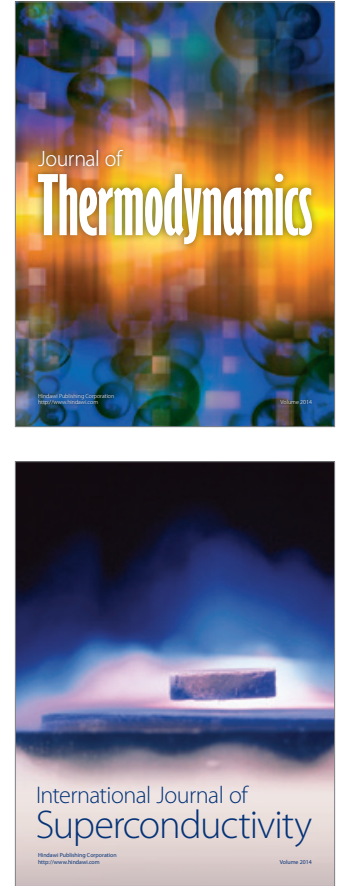
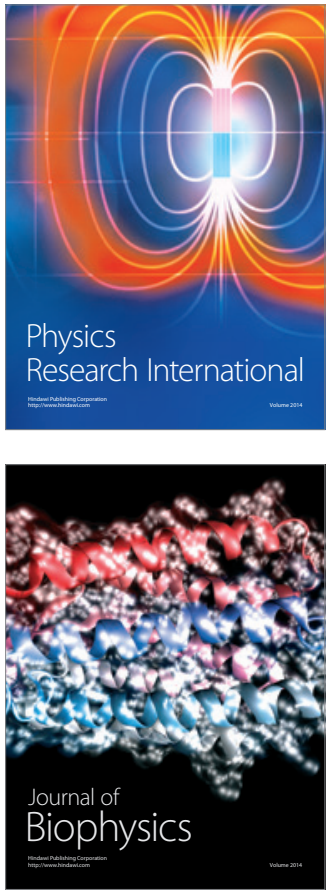
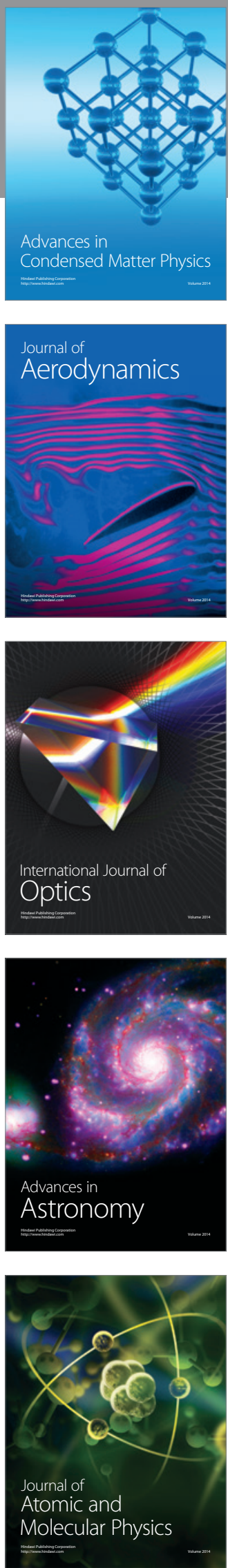